\begin{tabular}{|c|l|}
\hline Title & A nomalously long lifetimes of high-energy surface acoustic phonons \\
\hline Author(s) & Tamura, Shin-ichiro \\
\hline Citation & $\begin{array}{l}\text { Physical Review B, 30(2), 610-617 } \\
\text { https://doi.org/10.1103/PhysRevB.30.610 }\end{array}$ \\
\hline Issue Date & 1984-07-15 \\
\hline Doc URL & http://hdl.handle.net/2115/5953 \\
\hline Rights & Copyright $\odot$ 1984 A merican Physical Society \\
\hline Type & article \\
\hline File Information & PRB30-2.pdf \\
\hline
\end{tabular}

Instructions for use 


\title{
Anomalously long lifetimes of high-energy surface acoustic phonons
}

\author{
Shin-ichiro Tamura \\ Department of Engineering Science, Hokkaido University, Sapporo 060, Japan
}

(Received 3 January 1984)

\begin{abstract}
The lifetime of Rayleigh surface phonons as opposed to decay via anharmonic three-phonon processes is calculated in the regime $\hbar \omega_{R} \gg k_{B} T$. The surface phonon decays predominantly by combining with a bulk transverse phonon to create another bulk transverse phonon. The energymomentum conservation of the processes imposes the condition that those bulk phonons which may interact with the surface phonons should possess frequencies of the same order of $\omega_{R}$. However, such high-frequency phonons are rarely excited thermally under the condition considered here, leading to an anomalously long lifetime of the surface phonons in proportion to $\omega_{R}^{-4.5} T^{-0.5} \exp \left(A \hbar \omega_{R} / k_{B} T\right)$, where $A$ is a constant of the order of 0.1 . We also estimate numerically the magnitude of the lifetime.
\end{abstract}

\section{INTRODUCTION}

Lifetimes of surface acoustic phonons (Rayleigh waves) as opposed to decay via anharmonic three-phonon processes considered so far are restricted to those in the regime $\hbar \omega_{R} \lesssim k_{B} T$, where $\omega_{R}$ is the angular frequency of the surface phonons ( $R$ means the Rayleigh mode) and $T$ is the ambient temperature in a solid. ${ }^{1-4}$ It is well established both theoretically ${ }^{1}$ and experimentally ${ }^{5}$ that the decay rate (i.e., the reciprocal of lifetime) of surface phonons is proportional to $\omega_{R} T^{4}$ under the conditions $\hbar \omega_{R}<<k_{B} T$ and $\omega_{R} \tau_{\text {th }} \gg 1$, where $\tau_{\text {th }}$ is the relaxation time of thermally excited phonons which interact with surface phonons. It should be noted that this frequency and temperature dependence is identical to that of the attenuation rates of bulk phonons satisfying the same conditions. ${ }^{6} \mathrm{~A}$ slightly different result was deduced by Sakuma and Nakayama ${ }^{4}$ on the decay rate of surface phonons in the regime $\hbar \omega_{R} \simeq k_{B} T$. By numerical calculations they found that it is approximately described by $\omega_{R}^{3} T^{2}$.

The purpose of the present work is to discuss the lifetime of surface phonons of frequencies satisfying $\hbar \omega_{R} \gg k_{B} T$, which is motivated by the experiment conducted by Guo and Maris ${ }^{7}$ several years ago. They attempted to generate and detect, at low temperatures, surface heat pulses on a Si wafer, which are composed of high-frequency surface phonons. If they are detected, sharp focusing patterns of the surface phonons due to the elastic anisotropy of the crystal surface should be observed. Unfortunately, however, no signal was seen. The experiment was tried at temperatures of about $0.4 \mathrm{~K}$ where the condition $\hbar \omega \gg k_{B} T$ is well satisfied for phonons of frequencies higher than $50 \mathrm{GHz}$. Hence, we believe that the frequencies of most of the nonequilibrium surface phonons excited in their experiment may satisfy the above inequality. However, no theoretical analysis on the anharmonic decay of such high-energy surface phonons has been reported up to the present.

The lifetimes of bulk acoustic phonons in the regime $\hbar \omega \gg k_{B} T$ have been discussed extensively by Orbach and Vredevoe. ${ }^{8}$ According to their results transverse-acoustic
(TA) phonons possess an anomalously long lifetime, though longitudinal-acoustic (LA) phonons have a very short lifetime. This is because (unlike the case of LA phonons) the spontaneous two-phonon decays of the TA phonons are prohibited by energy and momentum conservation. We expect that a similar situation may hold for surface phonons, whose velocity $\left(v_{R}\right)$ is slower than that of the TA phonons $\left(V_{t}\right)$, e.g., typically $v_{R} \simeq 0.9 V_{t}$. By kinematical considerations we indeed find that surface phonons cannot decay into two phonons except through the collinear process $R \rightarrow R+R$ and that their decay necessitates the presence of thermally excited phonons with energy of the order of $\hbar \omega_{R}$. However, such highenergy phonons are rarely excited in the temperature regime we consider leading to the anomalously long lifetime of high-frequency surface phonons satisfying $\hbar \omega_{R} \gg k_{B} T$.

In the next section, we specify the phonon modes based on kinematical considerations in the elastic half-space which interact efficiently with the surface phonons. The formula for the lifetime of the surface phonons is derived and some numerical estimations of the lifetime are given in Sec. III. Throughout this work we employ the isotropic approximation. The continuum elasticity theory is also assumed and the acoustic dispersion due to the discreteness of the lattice is neglected. This is because we are interested in surface phonons of frequencies of about $100 \mathrm{GHz}$, that correspond to a wavelength of $500 \AA$ for $\mathrm{Si}$, a length much longer than interatomic distances.

\section{KINEMATICAL CONSIDERATIONS}

In this section we shall study energy and momentum conservation imposed kinematically on three-phonon processes and specify the phonon modes and spatial configuration of the interaction which contribute predominantly to the decay of the surface phonons. To begin, let us consider the process in which a surface phonon specified by $\left(\overrightarrow{\mathrm{q}}_{R}, \omega_{R}\right)$ combines with a thermally excited bulk phonon $\left(\vec{q}^{\prime}, \omega^{\prime}\right)$ to create a second phonon $\left(\vec{q}^{\prime \prime}, \omega^{\prime \prime}\right)$. Then denoting by $\vec{q}_{\|}$the component of the wave vector $\vec{q}$ parallel to the surface, the energy and momentum conservation can 
be written

$$
\begin{aligned}
& \omega_{R}+\omega^{\prime}=\omega^{\prime \prime}, \\
& \overrightarrow{\mathrm{q}}_{R}+\overrightarrow{\mathrm{q}}_{\|}^{\prime}=\overrightarrow{\mathrm{q}} \|{ }^{\prime \prime} .
\end{aligned}
$$

Next we introduce the velocity $v$ defined by $\omega=v\left|\overrightarrow{\mathrm{q}}_{\|}\right|=v q_{\|}\left(\right.$similarly, $\left.\omega_{R}=v_{R}\left|\overrightarrow{\mathrm{q}}_{R}\right|=v_{R} q_{R}\right)$. If we denote by $V$ a phase velocity of the bulk phonons (i.e., $V=V_{l}$ or $V_{t}$ ), we have

$$
V=v \sin \theta \text {, }
$$

where $\theta$ is the angle of the wave vector $\vec{q}$ measured from the surface normal and $v$ takes a value larger than $V_{t}$.

Now, eliminating $\overrightarrow{\mathrm{q}} \|$ from Eqs. (1) and (2), we have

$$
\begin{aligned}
\cos \Phi=\frac{v_{R} v^{\prime}}{\left(v^{\prime \prime}\right)^{2}}+\frac{1}{2\left(v^{\prime \prime}\right)^{2}}[ & {\left[v_{R}^{2}-\left(v^{\prime \prime}\right)^{2}\right] \frac{q_{R}}{q_{\|}^{\prime}} } \\
& \left.+\left[\left(v^{\prime}\right)^{2}-\left(v^{\prime \prime}\right)^{2}\right] \frac{q_{\|}^{\prime}}{q_{R}}\right],
\end{aligned}
$$

where $\Phi$ is the angle between $\overrightarrow{\mathrm{q}}_{R}$ and $\overrightarrow{\mathrm{q}}_{\|}^{\prime}$. The condition $\hbar \omega_{R} \gg k_{B} T$ implies $\omega_{R} \gg \omega^{\prime}$ for almost all phonons $\left(\overrightarrow{\mathrm{q}}^{\prime}, \omega^{\prime}\right)$ excited thermally at a temperature $T$ of a medium. Hence

$$
\frac{q_{\|}^{\prime}}{q_{R}} \ll \frac{v_{R}}{v^{\prime}}<1,
$$

and we may approximate Eq. (4) as

$$
\cos \Phi=\frac{1}{\left(v^{\prime \prime}\right)^{2}}\left[v_{R} v^{\prime}+\frac{1}{2}\left[v_{R}^{2}-\left(v^{\prime \prime}\right)^{2}\right] \frac{q_{R}}{q_{\|}^{\prime}}\right] .
$$

The right-hand side of Eq. (6) is now much less than -1 and accordingly the scattering of surface phonons is practically impossible. Energy and momentum conservation requires that the surface phonons are scattered only by phonons with $q_{\|}^{\prime} \simeq q_{R}$. However, such phonons are excited insignificantly under the present condition and therefore the lifetime of the surface phonons will become anomalously long.

From Eq. (4) or (6) we can specify the modes of phonons which may interact efficiently with the surface phonons in question. Here we note that the bulk phonons other than the surface phonons in the half-space are classified into two groups by their velocity spectra, one with $v \geq V_{t}$ and the other $v \geq V_{l}$, where $v_{R} \simeq 0.9 V_{t} \simeq 0.5 V_{l}$. To minimize $q_{R} / q_{\mid}^{\prime}=\left(v^{\prime} / v_{R}\right)\left(\omega_{R} / \omega^{\prime}\right)$ in Eq. (6), smaller values of $v^{\prime}$ are favorable. Furthermore, to minimize the modulus of the prefactor $\left[v_{R}^{2}-\left(v^{\prime \prime}\right)^{2}\right] /\left(v^{\prime \prime}\right)^{2}, v^{\prime \prime} \simeq V_{t}$ should be most desirable. ${ }^{9}$ Therefore, the predominant contribution to the interaction comes from the processes in which a surface phonon collides with a surface phonon or a bulk TA phonon propagating nearly parallel to the surface and as a result produces a TA phonon which travels also in the vicinity of the surface.

In the isotropic elastic medium occupying the halfspace, there exist two kinds of phonon modes which have the velocity spectrum $v \geq V_{t}{ }^{10,11}$ The first mode $\left(T_{\|}\right.$ mode) consists of TA phonons polarized parallel to the surface, and its range of velocity $v$ is $V_{t}$ to infinity de- pending on the angle of incidence of $\theta$ to the surface of $\vec{q}$. The second mode ( $T_{\perp}$ mode) consists of TA phonons polarized in the saggital plane followed by an evanescent pseudo-surface-wave. The velocity of this mode is confined in the finite range $V_{t} \leq v \leq V_{l}$. In the limit of $v \rightarrow V_{t}$, both modes are reduced to bulk TA phonons traveling parallel to the surface, and the evanescent component of the $T_{\perp}$ mode vanishes in this limit. However, there is an important difference between these two modes in regard to the depth dependence of the displacement vector $\overrightarrow{\mathrm{u}}$ of the medium. In order to satisfy the boundary condition at the free flat surface, as $v \rightarrow V_{t}$,

$$
\begin{aligned}
& \overrightarrow{\mathrm{u}}_{||}(z) \propto \cos \left(\sigma q_{||} z\right), \\
& u_{3}(z)=0,
\end{aligned}
$$

for the $T_{\|}$mode and

$$
\begin{aligned}
& \overrightarrow{\mathrm{u}}_{\|}(z)=\overrightarrow{0} \\
& u_{3}(z) \propto \sin \left(\sigma q_{\|} z\right),
\end{aligned}
$$

for the $T_{\perp}$ mode, where

$$
\sigma=\left[\left[\frac{v}{V_{t}}\right]^{2}-1\right]^{1 / 2}
$$

and we have fixed the Cartesian coordinate system so that the medium occupies the half-space $x_{3}=z \geq 0$ with the free flat surface parallel to the $\overrightarrow{\mathrm{x}}_{\|}=\left(x_{1}, x_{2}\right)=(x, y)$ plane. From Eqs. (7) and (8) we see that the $T_{\|}$mode has a finite amplitude at the surface, but the displacement vector of the $T_{\perp}$ mode vanishes there. Because the surface phonons are associated with lattice vibrations localized in a wavelength from the surface and furthermore $q_{\|} \simeq q_{R}$ for phonons which interact with the surface phonons, it is the $T_{\|}$mode that should contribute effectively to the decay of the surface phonons. This is valid even when the interaction is described through the strain fields $\xi_{i j}=\partial_{j} u_{i}$, because $\sigma$ also vanishes in the limit of $v \rightarrow V_{t}$.

\section{CALCULATION OF LIFETIME}

When the atoms of a solid are displaced from their equilibrium positions, the local potential energy in the solid may be expanded in the long-wavelength approximation as follows:

$$
W=\frac{1}{2 !} c_{i j k l} \eta_{i j} \eta_{k l}+\frac{1}{3 !} c_{i j k l m n} \eta_{i j} \eta_{k l} \eta_{m n}+O\left(\eta^{4}\right)
$$

where $\overleftrightarrow{\eta}$ is the deformation tensor defined by

$$
\eta_{i j}=\frac{1}{2}\left(\zeta_{i j}+\zeta_{j i}+\zeta_{k i} \zeta_{k j}\right),
$$

and the summation convention over repeated indices is assumed. In the isotropic approximation, the second-order and third-order elastic constants $c_{i j k l}$ and $c_{i j k l m n}$ which form the components of the fourth-rank and sixth-rank tensors, respectively, are written in terms of Lamé constants $\lambda, \mu$, and three other constants $\alpha, \beta$, and $\gamma$ as 


$$
\begin{aligned}
c_{i j k l}= & \lambda \delta_{i j} \delta_{k l}+\mu\left(\delta_{i k} \delta_{j l}+\delta_{i l} \delta_{j k}\right), \\
c_{i j k l m}= & \alpha \delta_{i j} \delta_{k l} \delta_{m n}+\beta\left[\delta_{i j}\left(\delta_{k m} \delta_{l n}+\delta_{k n} \delta_{l m}\right)+\delta_{k l}\left(\delta_{i m} \delta_{j n}+\delta_{i n} \delta_{j m}\right)+\delta_{m n}\left(\delta_{i k} \delta_{j l}+\delta_{i l} \delta_{j k}\right)\right] \\
& +\gamma\left[\left(\delta_{i k} \delta_{l m}+\delta_{i l} \delta_{k m}\right) \delta_{n j}+\left(\delta_{j k} \delta_{l m}+\delta_{j l} \delta_{k m}\right) \delta_{n i}+\left(\delta_{i k} \delta_{l n}+\delta_{i l} \delta_{k n}\right) \delta_{m j}+\left(\delta_{j k} \delta_{l n}+\delta_{j l} \delta_{k n}\right) \delta_{m i}\right] .
\end{aligned}
$$

Substituting Eqs. (12) and (13) into (10) and retaining the cubic terms in the particle displacement, we have

$$
W \rightarrow W_{3}=\frac{1}{2}(\beta+\lambda) \zeta_{i i} \zeta_{j k} \zeta_{j k}+(\gamma+\mu) \zeta_{i j} \zeta_{k i} \zeta_{k j}+\frac{\alpha}{6} \zeta_{i i} \zeta_{j j} \zeta_{k k}+\frac{\beta}{2} \zeta_{i i} \zeta_{j k} \zeta_{k j}+\frac{\gamma}{3} \zeta_{i j} \zeta_{j k} \zeta_{k i}
$$

The total potential energy due to cubic anharmonic terms in the half-space is then obtained by integrating $W_{3}$ over $z \geq 0$,

$$
W_{\text {tot }}=\int_{z \geq 0} W_{3}(\overrightarrow{\mathrm{r}}) d \overrightarrow{\mathrm{r}}
$$

where $\overrightarrow{\mathrm{r}}=\left(\overrightarrow{\mathrm{x}}_{\|}, x_{3}\right)=(x, y, z)$.

In the elastic half-space with a free flat surface, the displacement vector can be expanded as ${ }^{11}$

$\overrightarrow{\mathrm{u}}(\overrightarrow{\mathrm{r}})=\sum_{J}\left[\frac{\hbar}{2 \rho \omega_{J}}\right]^{1 / 2}\left\{a_{J} \overrightarrow{\mathrm{U}}^{J}(\overrightarrow{\mathbf{r}})+a_{J}^{\dagger}\left[\overrightarrow{\mathrm{U}}^{J}(\overrightarrow{\mathbf{r}})\right]^{*}\right\}$,

where $\rho$ is the mass density of the medium and $J=\left(\overrightarrow{\mathrm{q}}_{\|}, v, M\right)$ is a set of quantum numbers in which $M$ specifies the propagation modes of elastic waves both surface and bulk in character. The sum over $J$ is to be understood to be

$$
\begin{aligned}
\sum_{J} f(J)=\sum_{\overrightarrow{\mathrm{q}}_{\|}}\left(f\left(\overrightarrow{\mathrm{q}}_{\|}=\overrightarrow{\mathrm{q}}_{R}, v_{R}, R\right)\right. \\
\left.\quad+\sum_{M \neq R} \int_{D_{M}} \frac{d v}{v} f\left(\overrightarrow{\mathrm{q}}_{\|}, v, M\right)\right),
\end{aligned}
$$

where $D_{M}$ denotes the spectral range of the velocity $v$ for the bulk mode $M$. In Eq. (16), $a_{J}$ and its Hermitian conjugate $a_{J}^{\dagger}$ are the annihilation and creation operators of the phonons in the half-space, which commute with each other except for the case

$$
\left[a_{J}, a_{J^{\prime}}^{\dagger}\right]=\delta_{J, J^{\prime}}, \quad \delta_{J, J^{\prime}}=\delta_{\overrightarrow{\mathrm{q}}_{\|}, \overrightarrow{\mathrm{q}}_{\|}^{\prime}} \delta_{v, v^{\prime}} \delta_{M, M^{\prime}}
$$

If $v$ and $v^{\prime}$ belong to continuous spectra, we understand $\delta_{v, v^{\prime}}$ as $\delta_{v, v^{\prime}}=v \delta\left(v-v^{\prime}\right)$. Because the medium is assumed to be homogeneous in the directions parallel to the surface, the $\vec{x}_{\|}$dependence of $\vec{U}^{J}(\vec{r})$ should be described by a plane wave, i.e.,

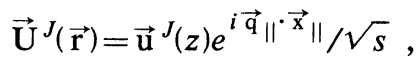

where $S$ is the surface area.

Now, the substitution of Eqs. (16) and (19) into (15) yields the anharmonic interaction Hamiltonian which causes three-phonon processes in the half-space. Then, rewriting the left-hand side of Eq. (15) as $\mathrm{H}_{3}$, we have

$$
\begin{aligned}
H_{3}=\left(\frac{\hbar}{2 \rho}\right]^{3 / 2} \frac{1}{\sqrt{S}} \sum_{J, J, J^{\prime \prime}} \frac{1}{\left(\omega_{J} \omega_{J^{\prime}} \omega_{J^{\prime \prime}}\right)^{1 / 2}} & {\left[\left(a_{J} a_{J^{\prime}} a_{J^{\prime \prime}}^{\dagger} F_{J J^{\prime} \bar{J}^{\prime \prime}}+a_{J^{\prime}}^{\dagger} a_{J^{\prime}}^{\dagger} a_{J^{\prime \prime}} F_{\bar{J}^{\prime} \bar{J}^{\prime \prime}}\right) \delta_{\overrightarrow{\mathrm{q}}_{\|}+\overrightarrow{\mathrm{q}}_{\|}^{\prime}, \overrightarrow{\mathrm{q}}_{\|}^{\prime \prime}}\right.} \\
& +\left(a_{J} a_{J^{\prime}}^{\dagger} a_{J^{\prime \prime}} F_{J \bar{J}^{\prime} J^{\prime \prime}}+a_{J^{\prime}}^{\dagger} a_{J^{\prime}} a_{J^{\prime \prime}}^{\dagger} F_{\bar{J}^{\prime} \bar{J}^{\prime \prime}}\right) \delta_{\overrightarrow{\mathrm{q}}_{\|}+\overrightarrow{\mathrm{q}}_{\|}^{\prime \prime}, \overrightarrow{\mathrm{q}}_{\|}^{\prime}} \\
& +\left(a_{J}^{\dagger} a_{J^{\prime}} a_{J^{\prime \prime}} F_{\bar{J}^{\prime} J^{\prime \prime}}+a_{J} a_{J^{\prime}}^{\dagger} a_{J^{\prime \prime}}^{\dagger} F_{J \bar{J}^{\prime} \bar{J}^{\prime \prime}}\right) \delta_{\overrightarrow{\mathrm{q}}_{\|,}, \overrightarrow{\mathrm{q}}_{\|}^{\prime}+\overrightarrow{\mathrm{q}}\|\|^{\prime \prime}},
\end{aligned}
$$

where

$$
\begin{aligned}
F_{J J^{\prime} \bar{J}^{\prime \prime}}=\int_{0}^{\infty} d z[ & \frac{1}{2}(\beta+\lambda) \xi_{i i}^{J}(z) \xi_{j k}^{J^{\prime}}(z)\left[\xi_{j k}^{J^{\prime \prime}}(z)\right]^{*}+(\gamma+\mu) \xi_{i j}^{J}(z) \xi_{k i}^{J^{\prime}}(z)\left[\xi_{k j}^{J^{\prime \prime}}(z)\right]^{*} \\
& \left.+\frac{\alpha}{6} \xi_{i i}^{J}(z) \xi_{j j}^{J^{\prime}}(z)\left[\xi_{k k}^{J^{\prime \prime}}(z)\right]^{*}+\frac{\beta}{2} \xi_{i i}^{J}(z) \xi_{j k}^{J^{\prime}}(z)\left[\xi_{k j}^{J^{\prime \prime}}(z)\right]^{*}+\frac{\gamma}{3} \xi_{i j}^{J}(z) \xi_{j k}^{J^{\prime}}(z)\left[\xi_{k i}^{J^{\prime \prime}}(z)\right]^{*}\right),
\end{aligned}
$$

etc., and here we have introduced $\xi_{i j}^{J}$ defined by

$$
\partial_{j}\left[u_{i}^{J}(z) e^{i \overrightarrow{\mathrm{q}}_{\|} \cdot \overrightarrow{\mathrm{x}}_{\|}}\right] \equiv \xi_{i j}^{J}(z) e^{i \overrightarrow{\mathrm{q}}_{\|} \cdot \overrightarrow{\mathrm{x}}_{\|}} .
$$

In deriving Eq. (20), the terms containing aaa and $a^{\dagger} a^{\dagger} a^{\dagger}$ which do not contribute to the energy-conserving processes are omitted. 
The lowest-order perturbation theory tells us that the lifetime $\tau_{J}$ of the phonon $J$ via three-phonon processes takes the form

$$
\begin{aligned}
& \tau_{J}^{-1}=\frac{\pi \hbar}{8 \rho^{3} \omega_{J} S} \sum_{J^{\prime}, J^{\prime \prime}} \frac{1}{\omega_{J^{\prime}} \omega_{J^{\prime \prime}}}\left[2\left(n_{J^{\prime}}-n_{J^{\prime \prime}}\right) G_{J J^{\prime} J^{\prime \prime}} \delta_{\overrightarrow{\mathrm{q}}_{\|}+\overrightarrow{\mathrm{q}}_{\|,}^{\prime}, \overrightarrow{\mathrm{q}}{ }_{\|}^{\prime \prime}} \delta\left(\omega_{J}+\omega_{J^{\prime}}-\omega_{J^{\prime \prime}}\right)\right.
\end{aligned}
$$

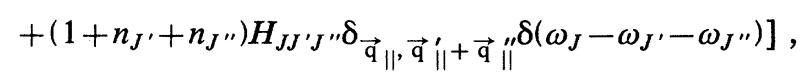

where

$$
\begin{aligned}
& G_{J J^{\prime} J^{\prime \prime}}=\left|F_{J J^{\prime} \bar{J}^{\prime \prime}}+F_{J^{\prime} J \bar{J}^{\prime \prime}}+F_{J \bar{J}^{\prime \prime} J^{\prime}}+F_{J^{\prime} \bar{J}^{\prime \prime} J}+F_{\bar{J}^{\prime \prime} J J^{\prime}}+F_{\bar{J}^{\prime \prime} J^{\prime} J}\right|^{2}, \\
& H_{J J^{\prime} J^{\prime \prime}}=\left|F_{J \bar{J}^{\prime} \bar{J}^{\prime \prime}}+F_{\bar{J}^{\prime} J \bar{J}^{\prime \prime}}+F_{J \bar{J}^{\prime \prime} \bar{J}^{\prime}}+F_{\bar{J}^{\prime} \bar{J}^{\prime \prime} J}+F_{\bar{J}^{\prime \prime} J \bar{J}^{\prime}}+F_{\bar{J}^{\prime \prime} \bar{J}^{\prime} J}\right|^{2},
\end{aligned}
$$

and $n_{J}$ stands for the Planckian distribution function in the thermal equilibrium, i.e.,

$$
n_{J}=\left[\exp \left(\hbar \omega_{J} / k_{B} T\right)-1\right]^{-1} \text {. }
$$

We apply these formulas to the decay of the surface phonons $J=\left(\vec{q}_{R}, v_{R}, R\right)$. In this case, as we have remarked in the preceding sections, the second term in square brackets of Eq. (23) which expresses the spontaneous decay into twophonon states is neglected. Taking account of the fact that $\hbar \omega_{R} \gg k_{B} T$, the lifetime $\tau\left(\omega_{R}\right)$ of the surface phonons becomes

$$
\begin{gathered}
\tau^{-1}\left(\omega_{R}\right)=\frac{\pi \hbar}{4 \rho^{3} \omega_{R} S} \sum_{M^{\prime}, M^{\prime \prime}} \sum_{\overrightarrow{\mathrm{q}}_{\|}^{\prime}} \int_{D_{M^{\prime}}} \frac{d v^{\prime}}{v^{\prime}} \int D_{M^{\prime \prime}} \frac{d v^{\prime \prime}}{v^{\prime \prime}} \frac{n\left[\omega\left(q_{\|}^{\prime}, v^{\prime}\right)\right]}{\omega\left(q_{\|}^{\prime}, v^{\prime}\right) \omega\left(\left|\overrightarrow{\mathrm{q}}_{R}+\overrightarrow{\mathrm{q}}_{\|}^{\prime}\right|, v^{\prime \prime}\right)} G\left(\overrightarrow{\mathrm{q}}_{R}, \overrightarrow{\mathrm{q}}_{\|}^{\prime} ; v_{R}, v^{\prime}, v^{\prime \prime}\right) \\
\times \delta\left(\omega_{R}+\omega\left(q_{\|}^{\prime}, v^{\prime}\right)-\omega\left(\left|\overrightarrow{\mathrm{q}}_{R}+\overrightarrow{\mathrm{q}}_{\|}^{\prime}\right|, v^{\prime \prime}\right)\right)
\end{gathered}
$$

where $G\left(\overrightarrow{\mathrm{q}}_{R}, \overrightarrow{\mathrm{q}}_{\|}^{\prime} ; v_{R}, v^{\prime}, v^{\prime \prime}\right)$ represents $G_{J J^{\prime} J^{\prime \prime}}$ with $\overrightarrow{\mathrm{q}} \|$ being replaced by $\overrightarrow{\mathrm{q}}_{R}+\overrightarrow{\mathrm{q}}_{\|}^{\prime}$ When the phonon mode $M_{\text {, }}\left(\boldsymbol{M}^{\prime}\right.$ or $\left.M^{\prime \prime}\right)$ represents the surface mode, $d v / v$ should be understood to be $d v \delta\left(v-v_{R}\right)$. The summation over $\overrightarrow{\mathrm{q}}_{\|}^{\prime}$ in Eq. (27) is transformed into two-dimensional integrals over polar variables $\left(q_{\|}^{\prime}, \phi\left(\vec{q}_{\|}^{\prime}\right)\right)$, where the angle $\phi$ is measured from the direction of $\overrightarrow{\mathrm{q}}_{R}$. The integration with respect to $\phi\left(\overrightarrow{\mathrm{q}}_{\|}^{\prime}\right)$ is readily carried out with the aid of the formula

$$
\int_{0}^{2 \pi} f(\cos \phi) \delta(a+b \cos \phi) d \phi=\frac{2}{\left(b^{2}-a^{2}\right)^{1 / 2}} \Theta\left(b^{2}-a^{2}\right) f(-a / b)
$$

where $\Theta(x)$ is the unit step function, i.e., $\Theta$ is unity if $x>0$ and vanishes otherwise. The result of this integration yields

$$
\tau^{-1}\left(\omega_{R}\right)=\frac{\hbar v_{R}}{8 \pi \rho^{3} \omega_{R}^{2}} \sum_{M^{\prime}, M^{\prime \prime}} \int_{D_{M^{\prime}}} \frac{d v^{\prime}}{\left(v^{\prime}\right)^{2}} \int_{D_{M^{\prime \prime}}} \frac{d v^{\prime \prime}}{\left(v^{\prime \prime}\right)^{3}} \int_{q_{\min }}^{q_{\max }} \frac{d q_{\|}^{\prime}}{q_{\|}^{\prime}} \frac{n\left[\omega\left(q_{\|}^{\prime}, v^{\prime}\right)\right]}{\sin \Phi} G\left(\overrightarrow{\mathrm{q}}_{R}, q_{\|}^{\prime}, \phi\left(\overrightarrow{\mathrm{q}}_{\|}^{\prime}\right)=\Phi ; v_{R}, v^{\prime}, v^{\prime \prime}\right),
$$

where

$$
\begin{aligned}
& q_{\max }= \begin{cases}\frac{v^{\prime \prime}-v_{R}}{v^{\prime}-v^{\prime \prime}} q_{R} \equiv \epsilon_{1}\left(v_{R}, v^{\prime}, v^{\prime \prime}\right) q_{R} & \text { for } v^{\prime}>v^{\prime \prime}, \\
\frac{v^{\prime \prime}+v_{R}}{v^{\prime \prime}-v^{\prime}} q_{R} \equiv \epsilon_{2}\left(v_{R}, v^{\prime}, v^{\prime \prime}\right) q_{R} & \text { for } v^{\prime}<v^{\prime \prime},\end{cases} \\
& q_{\min }=\frac{v^{\prime \prime}-v_{R}}{v^{\prime}+v^{\prime \prime}} q_{R} \equiv \epsilon_{0}\left(v_{R}, v^{\prime}, v^{\prime \prime}\right) q_{R},
\end{aligned}
$$

and $\Phi$ is defined by Eq. (4). The explicit expression of $\sin \Phi$ is given by

$$
\sin \Phi=\frac{\mid\left(v^{\prime}\right)^{2}-\left(v^{\prime \prime}\right)^{2} \perp}{2\left(v^{\prime \prime}\right)^{2} q_{R} q_{\|}^{\prime}}\left|\left(q_{\|}^{\prime}-\epsilon_{1} q_{R}\right)\left(q_{\|}^{\prime}-\epsilon_{2} q_{R}\right)\left[q_{\|}^{\prime}-\frac{\epsilon_{2}}{\epsilon_{1}} q_{\min }\right]\left(q_{\|}^{\prime}-q_{\min }\right)\right|^{1 / 2} .
$$

Now, $q_{\min } \simeq q_{R}$ and then also $\hbar \omega\left(q_{\|}^{\prime}, v^{\prime}\right) \gg k_{B} T$ holds. Therefore, in Eq. (29) the distribution function $n$ may be approximated as

$$
n\left[\omega\left(q_{\|}^{\prime}, v^{\prime}\right)\right] \simeq \exp \left(-\hbar v^{\prime} q_{\|}^{\prime} / k_{B} T\right) .
$$

Because $n$ decreases abruptly with increasing $q_{\|}^{\prime}$, we may write Eq. (29) as follows: 


$$
\begin{aligned}
& \tau^{-1}\left(\omega_{R}\right)=\frac{\hbar v_{R}}{4 \pi \rho^{3} \omega_{R}^{2}} \sum_{M^{\prime}, M^{\prime \prime}} \int_{D_{M^{\prime}}} \frac{d v^{\prime}}{\left(v^{\prime}\right)^{2}}\left[\int_{\substack{D_{M^{\prime \prime}} \\
v^{\prime \prime}<v^{\prime}}} \frac{d v^{\prime \prime}}{v^{\prime \prime}} \frac{1}{\left(v^{\prime}\right)^{2}-\left(v^{\prime \prime}\right)^{2}}\left(\frac{\epsilon_{1}}{\left(\epsilon_{1}-\epsilon_{2}\right)\left(\epsilon_{0}-\epsilon_{2}\right) \epsilon_{0}}\right]^{1 / 2}\right. \\
& \times G\left(\overrightarrow{\mathrm{q}}_{R}, q_{\min }, \phi\left(\overrightarrow{\mathrm{q}}_{\|}^{\prime}\right)=\pi ; v_{R}, v^{\prime}, v^{\prime \prime}\right) K\left(\epsilon_{1} q_{R}, q_{\min } ; v^{\prime}, T\right) \\
& +\int_{\substack{D_{M^{\prime \prime}} \\
v^{\prime \prime}>v^{\prime}}} \frac{1}{\left(v^{\prime \prime}\right)^{2}-\left(v^{\prime}\right)^{2}}\left(\frac{\epsilon_{1}}{\left(\epsilon_{1}-\epsilon_{2}\right)\left(\epsilon_{0}-\epsilon_{1}\right) \epsilon_{0}}\right)^{1 / 2} \\
& \left.\times G\left(\overrightarrow{\mathrm{q}}_{R}, q_{\min }, \phi\left(\overrightarrow{\mathrm{q}}_{\|}^{\prime}\right)=\pi ; v_{R}, v^{\prime}, v^{\prime \prime}\right) K\left(\epsilon_{2} q_{R}, q_{\min } ; v^{\prime}, T\right)\right],
\end{aligned}
$$

where

$K\left(q_{\max }, q_{\min } ; v^{\prime}, T\right)=\int_{q_{\min }}^{q_{\max }} \frac{\exp \left(-\hbar v^{\prime} q / k_{B} T\right)}{\left[\left(q_{\max }-q\right)\left(q-q_{\min }\right)\right]^{1 / 2}} d q=\pi \exp \left[-\hbar v^{\prime}\left(q_{\max }+q_{\min }\right) / 2 k_{B} T\right] I_{0}\left[\hbar v^{\prime}\left(q_{\max }-q_{\min }\right) / 2 k_{B} T\right]$.

In Eq. (35), $I_{0}$ is the modified Bessel function of zeroth order. Incidentally, note that $\Phi=\pi$ when $q_{\|}^{\prime}=q_{\mathrm{min}}$. The argument of $I_{0}$ is much larger than unity and we may use the formula for the asymptotic expansion of $I_{0}$, i.e.,

$$
I_{0}(x) \sim \frac{e^{x}}{(2 \pi x)^{1 / 2}}, x>1 .
$$

Then, we have

$$
K\left(q_{\max }, q_{\min } ; v^{\prime}, T\right)=\left[\frac{\pi k_{B} T}{\hbar v^{\prime}\left(q_{\max }-q_{\min }\right)}\right]^{1 / 2} \exp \left(-\hbar v^{\prime} q_{\min } / k_{B} T\right) .
$$

Substituting Eq. (37) into (34), it is deduced that

$$
\begin{gathered}
\tau^{-1}\left(\omega_{R}\right)=\frac{\hbar v_{R}}{8 \pi \rho^{3} \omega_{R}^{2}}\left[\frac{\pi k_{B} T}{2 \hbar q_{R}}\right]^{1 / 2} \sum_{M^{\prime}, M^{\prime \prime}} \int_{D_{M^{\prime}}} \frac{d v^{\prime}}{\left(v^{\prime}\right)^{2}} \int_{D_{M^{\prime \prime}}} \frac{d v^{\prime}}{\left(v^{\prime \prime}\right)^{2}}\left[\frac{1}{v^{\prime} v^{\prime \prime}\left(v^{\prime}+v_{R}\right) \epsilon_{0}}\right]^{1 / 2} G\left(\overrightarrow{\mathrm{q}}_{R}, q_{\min }, \phi\left(\overrightarrow{\mathrm{q}}_{\|}^{\prime}\right)=\pi ; v_{R}, v^{\prime}, v^{\prime \prime}\right) \\
\times \exp \left(-\hbar v^{\prime} q_{\min } / k_{B} T\right)
\end{gathered}
$$

In order to perform the integrations over $v^{\prime}$ and $v^{\prime \prime}$ we need the explicit expressions for $G$. As we have remarked in Sec. II, the thermal phonons which may scatter the nonequilibrium surface phonons effectively are the surface and $T_{\|}$ phonons. The depth dependence of the displacement vector for the surface phonons $J=\left(\overrightarrow{\mathrm{q}}_{R}, v_{R}, R\right)$ is given by ${ }^{10,11}$

$$
\begin{aligned}
& u_{j}^{J}(z)=i \frac{\left(q_{R}\right)_{j}}{q_{R}}\left[\frac{q_{R}}{Y}\right]^{1 / 2}\left[e^{-\delta q_{R} z}-\frac{2 \delta \eta}{1+\eta^{2}} e^{-\eta q_{R} z}\right], j=1,2 \\
& u_{3}^{J}(z)=-\delta\left(\frac{q_{R}}{Y}\right)^{1 / 2}\left[e^{-\delta q_{R} z}-\frac{2}{1+\eta^{2}} e^{-\eta q_{R} z}\right]
\end{aligned}
$$

where

$$
\delta=\left[1-\left[\frac{v_{R}}{V_{l}}\right]^{2}\right]^{1 / 2}, \quad \eta=\left[1-\left[\frac{v_{R}}{V_{t}}\right]^{2}\right]^{1 / 2}, \quad Y=(\delta-\eta)\left(\delta-\eta+2 \delta \eta^{2}\right) / 2 \delta \eta^{2}
$$

For $T_{\|}$phonons $J=\left(\overrightarrow{\mathrm{q}}_{\|}, v, T_{\|}\right)$, we have

$$
\begin{aligned}
& u_{1}^{J}(z)=-\frac{\left(q_{\|}\right)_{2}}{q_{\|}}\left[\frac{2 v^{2} q_{\|}}{\pi V_{t}^{2} \sigma}\right]^{1 / 2} \cos \left(\sigma q_{\|} z\right), \\
& u_{2}^{J}(z)=\frac{\left(q_{\|}\right)_{1}}{q_{\|}}\left(\frac{2 v^{2} q_{\|}}{\pi V_{t}^{2} \sigma}\right)^{1 / 2} \cos \left(\sigma q_{\|} z\right), \\
& u_{3}^{J}(z)=0,
\end{aligned}
$$


where $\sigma$ is defined by Eq. (9). Here we remark that the displacement vector of the $T_{\|}$phonons is orthogonal to those of other mode phonons in the elastic half-space polarized in the saggital plane and satisfies

$$
\begin{aligned}
& \int_{z>0}\left[\overrightarrow{\mathrm{U}}^{J}(\overrightarrow{\mathrm{r}})\right]^{*} \cdot \overrightarrow{\mathrm{U}}^{J^{\prime}}(\overrightarrow{\mathrm{r}}) d \overrightarrow{\mathrm{r}}=\delta_{J, J^{\prime}}, \\
& \int_{V_{t}}^{\infty} \overrightarrow{\mathrm{u}}^{J}(z) \cdot\left[\overrightarrow{\mathrm{u}}^{J}\left(z^{\prime}\right)\right]^{*} \frac{d v}{v}=\delta\left(z-z^{\prime}\right) .
\end{aligned}
$$

After straightforward but tedious calculations we find that for the process $R+R \rightarrow T_{\|}, G$ is proportional to $\sin ^{2} \phi$. However, $\phi=\pi$ yields the dominant contributions through energy and momentum conservation [cf., Eq. (38)] and this process is suppressed in comparison with the contribution from the process $R+T_{\|} \rightarrow T_{\|}$. For the latter process we obtain

$$
\begin{aligned}
& G\left(\overrightarrow{\mathrm{q}}_{R}, q_{\min }, \phi\left(\overrightarrow{\mathrm{q}}_{\|}^{\prime}\right)=\pi ; v_{R}, v^{\prime}, v^{\prime \prime}\right)= \frac{4\left(\delta v^{\prime} v^{\prime \prime}\right)^{2}\left(\epsilon^{\prime} \epsilon^{\prime \prime}\right)^{3}}{\pi^{2} V_{t}^{4} \sigma^{\prime} \sigma^{\prime \prime} Y} \\
& \times q_{R}^{7}\left\{\left[(\beta+\lambda)\left(\frac{v_{R}}{V_{l}}\right)^{2}+2(\lambda+\mu)\right]\right. \\
& \times \frac{\delta^{2}+\left(\epsilon^{\prime} \sigma^{\prime}\right)^{2}+\left(\epsilon^{\prime \prime} \sigma^{\prime \prime}\right)^{2}}{\left[\delta^{2}+\left(\epsilon^{\prime} \sigma^{\prime}+\epsilon^{\prime \prime} \sigma^{\prime \prime}\right)^{2}\right]\left[\delta^{2}+\left(\epsilon^{\prime} \sigma^{\prime}-\epsilon^{\prime \prime} \sigma^{\prime \prime}\right)^{2}\right]} \\
&\left.-4(\lambda+\mu) \frac{\eta^{2}}{1+\eta^{2}} \frac{\eta^{2}+\left(\epsilon^{\prime} \sigma^{\prime}\right)^{2}+\left(\epsilon^{\prime \prime} \sigma^{\prime \prime}\right)^{2}}{\left[\eta^{2}+\left(\epsilon^{\prime} \sigma^{\prime}+\epsilon^{\prime \prime} \sigma^{\prime \prime}\right)^{2}\right]\left[\eta^{2}+\left(\epsilon^{\prime} \sigma^{\prime}-\epsilon^{\prime \prime} \sigma^{\prime \prime}\right)^{2}\right]}\right\}^{2}
\end{aligned}
$$

where

$$
\sigma^{\prime}=\left[\left(\frac{v^{\prime}}{V_{t}}\right]^{2}-1\right]^{1 / 2}, \sigma^{\prime \prime}=\left[\left[\frac{v^{\prime \prime}}{V_{t}}\right]^{2}-1\right]^{1 / 2},
$$

and we set $\epsilon_{0}=\epsilon^{\prime}$ and $1-\epsilon_{0}=\epsilon^{\prime \prime}$ for the sake of convenience. In deriving Eq. (44), we have retained terms of the form of

$$
\int_{0}^{\infty} d z e^{-\delta q_{R} z} \cos \left(\sigma^{\prime} q_{||}^{\prime} z\right) \cos \left(\sigma^{\prime \prime} q_{\|\|^{\prime \prime}}\right)
$$

in Eq. (22) and neglected the contributions of integrals which involve sinusoidal functions which vanish at the surface. In the curly brackets of Eq. (44), the term proportional to the third-order elastic constant $\alpha$ does not appear because $\xi_{i i}^{J}=0$ for TA phonons and the term proportional to $\gamma$ is suppressed because it depends on $\sin ^{2} \phi(\phi \simeq \pi)$. Accordingly, the contribution of this process to the decay rate is

$$
\begin{aligned}
& \tau^{-1}\left(\omega_{R}\right)= \frac{\hbar \delta^{2} \omega_{R}^{5}}{2 \pi^{3} \rho^{3} v_{R}^{6} V_{t}^{4} Y}\left(\frac{\pi k_{B} T}{2 \hbar q_{R}}\right]^{1 / 2} \\
& \times \int_{V_{t}}^{\infty} \frac{d v^{\prime}}{\sigma^{\prime}} \int_{V_{t}}^{\infty} \frac{d v^{\prime \prime}}{\sigma^{\prime \prime}}\left(\epsilon^{\prime} \epsilon^{\prime \prime}\right)^{5 / 2}\left(\frac{1}{v^{\prime} v^{\prime \prime}\left(v^{\prime}+v^{\prime \prime}\right)}\right]^{1 / 2} \\
& \times\left\{\left[(\beta+\lambda)\left(\frac{v_{R}}{V_{l}}\right]^{2}+2(\lambda+\mu)\right] \frac{\delta^{2}+\left(\epsilon^{\prime} \sigma^{\prime}\right)^{2}+\left(\epsilon^{\prime \prime} \sigma^{\prime \prime}\right)^{2}}{\left[\delta^{2}+\left(\epsilon^{\prime} \sigma^{\prime}+\epsilon^{\prime \prime} \sigma^{\prime \prime}\right)^{2}\right]\left[\delta^{2}+\left(\epsilon^{\prime} \sigma^{\prime}-\epsilon^{\prime \prime} \sigma^{\prime \prime}\right)^{2}\right]}\right. \\
&\left.\quad-4(\lambda+\mu) \frac{\eta^{2}}{1+\eta^{2}} \frac{\eta^{2}+\left(\epsilon^{\prime} \sigma^{\prime}\right)^{2}+\left(\epsilon^{\prime \prime} \sigma^{\prime \prime}\right)^{2}}{\left[\eta^{2}+\left(\epsilon^{\prime} \sigma^{\prime}+\epsilon^{\prime \prime} \sigma^{\prime \prime}\right)^{2}\right]\left[\eta^{2}+\left(\epsilon^{\prime} \sigma^{\prime}-\epsilon^{\prime \prime} \sigma^{\prime \prime}\right)^{2}\right]}\right\}^{2} \\
& \times \\
& \times
\end{aligned}
$$

where

$$
A=\epsilon_{0} v^{\prime} / v_{R} .
$$

From this equation, we find that the lifetime of the surface phonons is very long as indicated by the presence of the exponential factor of the form of $\exp \left(-A \hbar \omega_{R} / k_{B} T\right)$.

In order to estimate the order of magnitude of the lifetime, we convert the integrals over $v^{\prime}$ and $v^{\prime \prime}$ of Eq. (47) into integrals over $\sigma^{\prime}$ and $\sigma^{\prime \prime}$ by the relation

$$
d v=V_{t} \frac{\sigma}{\sigma^{2}+1} d \sigma .
$$


With increasing $\sigma^{\prime}$ and $\sigma^{\prime \prime}$ the factor $A$ varies only slowly, whereas the integrand other than the exponential factor decreases rather rapidly. Therefore, we may approximate $A$ to be its value at $\sigma^{\prime}=\sigma^{\prime \prime}=0$ (or $v^{\prime}=v^{\prime \prime}=V_{t}$ ), i.e.,

$$
A \rightarrow A_{0}=\frac{V_{t}-v_{R}}{2 v_{R}},
$$

and remove the exponential factor from the integrand. Note that $A_{0}$ is the minimum value of $A$ of the order of 0.1 . With this approximation, we finally obtain the expression for the decay rate,

$$
\tau^{-1}\left(\omega_{R}\right)=\frac{\hbar \delta^{2} \omega_{R}^{5}}{2 \pi^{3} \rho^{3} v_{R}^{11 / 2} V_{t}^{7 / 2} Y}\left(\frac{\pi k_{B} T}{2 \hbar \omega_{R}}\right)^{1 / 2} \exp \left(-A_{0} \hbar \omega_{R} / k_{B} T\right) \tilde{I}
$$

where

$$
\begin{aligned}
\widetilde{I}=\int_{0}^{\infty} \frac{d \sigma^{\prime}}{\left[\left(\sigma^{\prime}\right)^{2}+1\right]^{3 / 4}} \int_{0}^{\infty} & \frac{d \sigma^{\prime \prime}}{\left[\left(\sigma^{\prime \prime}\right)^{2}+1\right]^{3 / 4}}\left(\epsilon^{\prime} \epsilon^{\prime \prime}\right)^{5 / 2}\left[\frac{1}{\left[\left(\sigma^{\prime}\right)^{2}+1\right]^{1 / 2}+\left[\left(\sigma^{\prime \prime}\right)^{2}+1\right]^{1 / 2}}\right]^{1 / 2} \\
\times\left\{\left[(\beta+\lambda)\left[\frac{v_{R}}{V_{l}}\right]^{2}+2(\lambda+\mu)\right] \frac{\delta^{2}+\left(\epsilon^{\prime} \sigma^{\prime}\right)^{2}+\left(\epsilon^{\prime \prime} \sigma^{\prime \prime}\right)^{2}}{\left[\delta^{2}+\left(\epsilon^{\prime} \sigma^{\prime}+\epsilon^{\prime \prime} \sigma^{\prime \prime}\right)^{2}\right]\left[\delta^{2}+\left(\epsilon^{\prime} \sigma^{\prime}-\epsilon^{\prime \prime} \sigma^{\prime \prime}\right)^{2}\right]}\right. & ]^{2} . \\
& \left.-4(\lambda+\mu) \frac{\eta^{2}}{1+\eta^{2}} \frac{\eta^{2}+\left(\epsilon^{\prime} \sigma^{\prime}\right)^{2}+\left(\epsilon^{\prime \prime} \sigma^{\prime \prime}\right)^{2}}{\left[\eta^{2}+\left(\epsilon^{\prime} \sigma^{\prime}+\epsilon^{\prime \prime} \sigma^{\prime \prime}\right)^{2}\right]\left[\eta^{2}+\left(\epsilon^{\prime} \sigma^{\prime}-\epsilon^{\prime \prime} \sigma^{\prime \prime}\right)^{2}\right]}\right\}^{\prime}
\end{aligned}
$$

Using the numerical values $\beta=5.7 \times 10^{11}$ $\lambda=5.35 \times 10^{11} \quad \mathrm{dyn} / \mathrm{cm}^{2}, \quad \mu=6.86 \times 10^{11}$ dyn $/ \mathrm{cm}^{2}$, $V_{t}=5.42 \times 10^{5} \quad \mathrm{~cm} / \mathrm{sec}, \quad V_{l}=9.04 \times 10^{5} \mathrm{~cm} / \mathrm{sec}$, $v_{R}=4.96 \times 10^{5} \mathrm{~cm} / \mathrm{sec}$, and $\rho=2.33 \mathrm{~g} \mathrm{~cm}^{-3}$ for $\mathrm{Si}^{12}$ we have

$$
\tau^{-1}\left(\omega_{k}\right)=\widetilde{B} \omega_{R}^{9 / 2} T^{1 / 2} \exp \left(-\widetilde{A} \omega_{R} / T\right),
$$

where

$$
\begin{aligned}
& \widetilde{A}=3.5 \times 10^{-13} \sec \mathrm{K}, \\
& \widetilde{B}=2.7 \times 10^{-54} \sec ^{3.5} \mathrm{~K}^{-0.5} .
\end{aligned}
$$

For instance, at $T=0.4 \mathrm{~K}$ the lifetime of $100-\mathrm{GHz}$ surface phonons is

$$
\tau=4.8 \times e^{0.55}=8.3,
$$

in units of sec, and the corresponding mean free path becomes a macroscopic distance.

\section{CONCLUDING REMARKS}

Stimulated by the experiments of Guo and Maris, ${ }^{7}$ we have calculated the lifetime of high-energy surface phonons satisfying $\hbar \omega_{R} \gg k_{B} T$ and found that they are substantially stable against anharmonic decay. The formula obtained for the lifetime is similar to that of the bulk TA phonons satisfying the same condition but they are different from each other in details. Other than the power dependences on the frequency and temperature, the value of the coefficient $A_{0}(\simeq 0.05)$ in Eq. (50) is 1 order of magnitude smaller than that of the bulk TA phonons for which $A_{0} \simeq 0.3 .^{8}$ This is due to the fact that the velocity of the bulk TA phonons which interact predominantly with the surface phonons is very close to $v_{R}\left(v_{R} \simeq 0.9 V_{t}\right)$, whereas the velocity $V_{l}$ of the LA phonons into which the TA phonons decay satisfies $V_{t} \simeq V_{l} / \sqrt{3}$.

Through this work, it is concluded that the otherwise unavoidable intrinsic damping of the surface phonons due to anharmonic interaction essentially does not work in the regime $\hbar \omega_{R} \gg k_{B} T$. Our calculations are based on the isotropic approximations. Real crystalline solids are anisotropic in general. However, similar to the case for the decay of bulk TA phonons, the conclusion we have obtained here would be likely to be applied to the decay of the surface phonons in anisotropic solids. This is because the spontaneous decay of a phonon by anharmonic processes into a set of phonons of higher-phase velocity is prohibited by the energy-momentum conservation even in anisotropic crystals. ${ }^{13}$

Besides the anharmonic interaction, the propagation of surface phonons is influenced severely by the presence of various elastic inhomogeneities localized in the vicinity of the surface. The scattering of surface phonons by these inhomogeneities is highly frequency dependent. ${ }^{14-17}$ For instance, the frequency dependence of the attenuation rate due to scattering by surface roughness is proportional to $\omega_{R}^{5} f\left(\omega_{R}\right)$, where $f \rightarrow 1$ as $\omega_{R} \rightarrow 0$ but $f \simeq \omega_{R}^{-1}$ for $\omega_{R} \gg v_{R} / a$ ( $a$ is the correlation length of the roughness). ${ }^{17}$ According to the formula of Maradudin and Mills ${ }^{17}$ the mean free path of about $10^{-6} \mathrm{~cm}$ is estimated for $100-\mathrm{GHz}$ surface phonons propagating on a surface with the amplitude of roughness of $100 \AA$ (also for $a=100 \AA$ ). Hence, a very flat surface, e.g., one flat on the atomic scale, is required to observe the ballistic propagation of high-frequency surface phonons of about 100 $\mathrm{GHz}$.

\section{ACKNOWLEDGMENTS}

The author is indebted to Professor H. J. Maris for conversations about the details of his experiment. He is also grateful to Professor T. Sakuma for a helpful comment on the manuscript. This work is partially supported by the Grant-in-Aid for Scientific Research from the Ministry of Education, Science and Culture, Japan. 
${ }^{1}$ A. A. Maradudin and D. L. Mills, Phys. Rev. 173, 881 (1968).

${ }^{2}$ H. J. Maris, Phys. Rev. 188, 1308 (1969).

${ }^{3}$ P. J. King and F. W. Sheard, Proc. Roy. Soc. London, Ser. A 320, 175 (1970).

${ }^{4}$ T. Sakuma and T. Nakayama, Appl. Phys. Lett. 25, 176 (1974).

${ }^{5}$ E. Salzmann, T. Plieninger, and K. Dransfeld, Appl. Phys. Lett. 13, 14 (1968).

${ }^{6}$ For a review, see H. J. Maris, Physical Acoustics, edited by W. P. Mason and R. N. Thurston (Academic, New York, 1971), Vol. 8 p. 279.

${ }^{7}$ C.-J. Guo and H. J. Maris (unpublished); see also H. J. Maris (unpublished).

${ }^{8}$ R. Orbach and L. A. Vredevoe, Physics (N.Y.) 1, 92 (1964).

${ }^{9}$ When $v^{\prime \prime}=v_{R^{\prime}}, \cos \Phi=v^{\prime} / v_{R}$. This means that three-surfacephonon processes are possible irrespective of the magnitude of $q_{\|}^{\prime} / q_{R}$. However, these processes are restricted to be col- linear ones and then we neglect this case.

${ }^{10} \mathrm{~W}$. M. Ewing and W. S. Jardetzby, and F. Press, Elastic Waves in Layered Media (McGraw-Hill, New York, 1957).

${ }^{11}$ H. Ezawa, Ann. Phys. (N.Y.) 67, 438 (1971).

${ }^{12}$ For the data of the third-order elastic constants of Si, see $\mathbf{J}$. Philip and M. A. Breazeale, J. App. Phys. 54, 752 (1983).

${ }^{13}$ M. Lax, P. Hu, and V. Narayanamurti, Phys. Rev. B 23, 3095 (1981).

${ }^{14}$ R. G. Steg and P. G. Klemens, Phys. Rev. Lett., 24, 381 (1970).

${ }^{15}$ T. Nakayama and T. Sakuma, J. Appl. Phys. 46, 2445 (1975); 47, 2263 (1976).

${ }^{16}$ M. Narita, T. Sakuma, and T. Nakayama, J. Appl. Phys. 49, 5507 (1978).

${ }^{17}$ A. A. Maradudin and D. L. Mills, Ann. Phys. (N.Y.) 100, 262 (1976). 